\title{
Oracy Nogueira, entre São Paulo et Chicago
}

L'exploration des relations raciales

\section{Christophe Brochier}

\section{(2) OpenEdition}

\section{Journals}

Édition électronique

URL : http://journals.openedition.org/ress/900

DOI : $10.4000 /$ ress. 900

ISSN : 1663-4446

Éditeur

Librairie Droz

\section{Édition imprimée}

Date de publication : 15 octobre 2011

Pagination : 179-208

ISBN : 978-2-600-01551-6

ISSN : 0048-8046

\section{Référence électronique}

Christophe Brochier, "Oracy Nogueira, entre São Paulo et Chicago », Revue européenne des sciences sociales [En ligne], 49-1 | 2011, mis en ligne le 01 janvier 2015, consulté le 20 avril 2019. URL : http:// journals.openedition.org/ress/900; DOI : 10.4000/ress.900 


\title{
ORACY NOGUEIRA, ENTRE SÃO PAULO ET CHICAGO L'EXPLORATION DES RELATIONS RACIALES
}

CHRISTOPHE BROCHIER

CREDA (IHEAL) - Université Paris-VIII chrisbrochier@yahoo.com

\begin{abstract}
Résumé. Cet article se propose de décrire la carrière d'Oracy Nogueira, sociologue brésilien actif entre les années 1940 et les années 1960. Nogueira est essentiellement connu pour ses études sur les relations raciales documentant la discrimination raciale à l'égard des Noirs dans la région de São Paulo. Le texte revient sur sa carrière en expliquant l'influence qu'a eu sur lui sa formation au sein de l'École Libre de Sociologie et de Politique sous l'orientation de l'Américain Donald Pierson. On étudie également comment le séjour d'étude de Nogueira à Chicago a modifié sa façon d'envisager les relations raciales. Les enquêtes qu'il a produites par la suite sont en effet particulièrement originales. Elles combinent un grand attachement à la méthode ethnographique en même temps qu'une intention militante, opposée à celle de son mentor, et tournée vers la dénonciation des injustices raciales. En conclusion on examine la postérité de l'auteur dans le cadre des changements de perspectives au sujet du racisme.
\end{abstract}

Mots-clés: Brésil; histoire de la sociologie; relations raciales; ethnographie; école de Chicago

\begin{abstract}
This article describes the career of Oracy Nogueira, a Brazilian sociologist working in São Paulo during the 1940s, 1950s and 1960s. Nogueira is essentially known for his studies on racial relations informing the racial discrimination towards the Blacks. The text returns on its career by explaining the influence that its training within the Free School of Sociology and Politics under the orientation of the American Donald Pierson had on him. We also study how his stay in Chicago modified the way he looked at the racial relation problems. The inquiries which he produced afterward are indeed particularly original. They combine a strong commitment to the ethnographical method at the same time as a militant intention, opposite to that of his mentor, and turned to the denunciation of racial injustices. In conclusion we examine the actual reputation of the author within the framework of the changes of perspectives on the subject of racism.
\end{abstract}

Keywords: Brazil; racial relations; history of sociology; ethnography; chicago school of sociology 
La sociologie des populations noires est l'un des thèmes centraux et des plus polémiques de la sociologie brésilienne. Les débats ont porté sur l'identité nationale et les problèmes de la modernisation. Des intellectuels de différentes nationalités ont participé à ces discussions avec des approches parfois différentes. L'histoire de l'étude des relations raciales qui s'est imposée, notamment à la suite de Florestan Fernandes ${ }^{1}$ a parfois négligé l’analyse des intentions, des outils et des manières de travailler de ces différents contributeurs. Il en va ainsi du travail de l’Américain Donald Pierson, qui dans les années 1940 et 1950 a été l’un des chercheurs les plus importants au Brésil. Le courant de sociologie qu'il a importé s'inspirait directement de Robert Park, dont il avait été l'un des étudiants. En matière d'études des relations raciales ses travaux se sont notamment heurtés à ceux du Français Bastide et du Brésilien Florestan Fernandes (à São Paulo).

Une manière fertile d'étudier l'histoire de l'étude des relations raciales au Brésil à cette époque consiste ainsi à s'intéresser à la carrière de l'un des élèves de Pierson qui collabora également aux travaux de Bastide: Oracy Nogueira. Nogueira (1917-1996), dont l'œuvre connaît aujourd'hui un regain d'intérêt², fut l'un des chercheurs de terrain les plus doués de sa génération et le concepteur d'une interprétation personnelle, empiriquement fondée, de la «situation raciale » brésilienne. Étudiant à São Paulo puis à Chicago, Nogueira a produit des analyses sur les races s'inspirant d'idées scientifiques et de situations vécues dans les deux pays. L'étude attentive de la façon dont il a conduit ses recherches, nous permettra de comprendre certains aspects des conflits d'intérêts scientifiques et idéologiques en vigueur au Brésil dans les années 1940-1950 sur les races et le racisme. On insistera en particulier sur la façon dont Nogueira a essayé de concilier ses différentes influences sociologiques et ses préoccupations éthiques.

I Florestan Fernandes a été l'un des principaux sociologues brésiliens des années 1950-1960. Né en 1920 dans une famille pauvre de São Paulo, il a dû commencer à travailler très jeune. Après des études primaires et secondaires difficiles il parvient à être admis à l'Université de São Paulo (USP) au début des années 1940. II y deviendra l'un des meilleurs étudiants de Bastide qu'il remplacera au milieu des années 1950. Dans les années 1960, Fernandes est le tout puissant chef du département de sociologie avec des élèves appelés à former une génération particulièrement influente. La sociologie de la USP à cette époque est orientée vers l'analyse critique des évolutions de la société capitaliste brésilienne.

2 Voir notamment Maria Laura Cavalcanti (1995, 1996, 1998). 


\section{RAPIDE APERÇU DE LA SOCIOLOGIE À SÃO PAULO DANS LES ANNÉES 1940}

La sociologie est enseignée au Brésil dès les années 1920 dans les écoles normales et des manuels apparaissent dès les années 1930, mais les recherches sociologiques en tant que telles sont pratiquement inexistantes avant les années 1940³. Par ailleurs, bien qu’à Rio de Janeiro l'université du district fédéral fonctionne dès 1935 (Miceli, 1987) avec des enseignants prestigieux tels que Gilberto ${ }^{4}$ Freyre et Arthur Ramos ${ }^{5}$, c'est essentiellement du côté de São Paulo que se feront les avancées en matière de pratique de la sociologie. En effet, après la révolution régionale avortée de 1932, le riche état de São Paulo se dote de deux institutions accordant une grande importance aux sciences sociales: L’École Libre de Sociologie et de Politique (ELSP) en 1933 et la faculté des lettres de l’université de São Paulo (USP) en 1934. La première est financée (chichement) par le patronat et se fixe pour tâche de former des technocrates pour l'administration et les entreprises locales ${ }^{6}$. Elle dispense des cours de sociologie, psychologie, anthropologie à un public hétéroclite mais à qui on impose de savoir lire l’anglais. La deuxième institution est crée par les autorités de l'état

3 Sur les débuts de la sociologie au Brésil, avant et après les années 1930, on se verra notamment Candido (1959) et Schwartzman (1979).

4 Gilberto de Mello Freyre est sans doute le sociologue brésilien dont les œuvres ont été le plus lues. Né à Recife en 1900 dans une famille traditionnelle et influente, il étudie aux États-Unis au début des années 1920. II subit notamment l'influence de Boas et se convertit à l'anthropologie culturelle. II en tirera l'orientation intellectuelle de son premier et plus célèbre ouvrage en 1933, Casa grande e senzala (en français: «Maîtres et esclaves»). Cette brillante histoire de la société rurale traditionnelle du Nord du pays fait de lui à l'époque l'interprète le plus écouté mais également le plus polémique de la société brésilienne. Son oeuvre complète comporte plus de 20 ouvrages dressant un portrait complet de l'évolution de la société brésilienne du dix-septième jusqu'au vingtième siècle.

5 Arthur Ramos de Araújo Pereira a été dans les années 1930 et 1940 une figure centrale études afro-brésiliennes. Né en 1903 dans une petite ville de l'état rural d'Alagoas, fils de médecin, il étudie, lui aussi la médecine. II se spécialise dans la psychiatrie à l'université de Salvador et se tourne vers le cas des populations noires jugées alors inadaptées à la civilisation moderne. II enseigne ensuite les sciences sociales à Rio de Janeiro.

6 Au sujet de l'ELSP: Limongi (1989), Mendonza (2005), Miceli, (1987), Schwartzman (1979), et surtout Kantor et al. (200 I). 
de São Paulo afin d'enrichir l'offre de formation supérieure ${ }^{7}$. Alors que la USP va faire appel pour les sciences sociales à des Français (Paul-Arbousse Bastide puis Lévi-Strauss), l'ELSP va avoir recours à un personnel plus anglo-saxon; pour la sociologie, les Américains Horace Davis puis Samuel Lowrie ${ }^{8}$.

Dans les deux institutions, les débuts sont hésitants et les élèves difficiles à recruter. La recherche sociologique n'est donc pas tout de suite une priorité, bien que les professeurs étrangers, comme Lowrie ou Lévi-Strauss, souhaitent s'y consacrer. Dans les deux institutions l'activité sociologique va en fait prendre son essor dans les années 1940 grâce à Roger Bastide à l'Université et à Donald Pierson à l'École Libre. Ils vont former une génération de jeunes chercheurs à la recherche par un entraînement aux méthodes d'enquêtes et le suivi de travaux personnels pour le doctorat à la USP et le «master » à l'ELSP. Ainsi Bastide fait-il accomplir à ses étudiants dans les années 1940 de courts exercices d'observation et d'entretiens 9 et Pierson amène-t-il dès les premières années ses élèves sur le terrain pour des enquêtes mêlant observations spontanées, entretiens, histoires de vie, et observations participantes (Kantor et al.,200I, Valladares, 2006).

Mais les points communs entre ces deux institutions recouvrent également des divergences importantes. Si, à l’université de São Paulo, les enseignants sont d’accord sur l'importance de recueillir des données de première main pour décrire l'état du Brésil, leur sociologie est tournée vers les idées, la reconstitution historique et les sociologues théoriciens. À l’ELSP, en revanche, Pierson et Emilio Willems ${ }^{10}$ travaillent en utilisant les outils de la sociologie de Chicago

7 Sur les débuts de l'université de São Paulo, la littérature est vaste. On verra en particulier: Jackson (2007), Coelho (198I) et Cardoso (1987).

8 Davis ne resta que deux ans à l'ELSP en raison de ses idées politiques communistes qui n'étaient pas du goût des bailleurs de fonds. Lowrie travailla à L'ELSP jusqu'en 1939 et publia notamment des études statistiques sur les ouvriers. II s'intéressa également au cas des Noirs, d'un point de vue démographique.

9 Certains de ces travaux sont disponibles à l'IMEC de Caen.

10 Né en Allemagne en 1905, Emilio Willems avait étudié l'économie avant d'émigrer au Brésil en 1931. II enseigna ensuite les sciences sociales à l'ELSP à partir de 1936 puis l'anthropologie à la USP à partir des années 1940. En 1949 il quitte le Brésil pour s'installer aux États-Unis. II enseigne alors à Venderbilt. II peut être considéré comme l'un des pionniers de la sociologie et de l'anthropologie modernes au Brésil. 
appliqués à l'étude de la coexistence entre les groupes sociaux (Vila-Nova, 1998). De même, pour eux, les études de communauté constituent un but en soi : il s'agit avant tout de combler le déficit de faits concernant un Brésil en voie de modernisation. Dans les années I940-1950, l'équipe de l’ELSP se distinguera par des ouvrages importants ${ }^{11}$ et la publication régulière de la revue Sociologia.

À ces divergences se superpose une sorte de rivalité des deux institutions pour attirer les rares «bons » étudiants capables de maîtriser les langues étrangères. L’École Libre de Sociologie et de Politique offre une formation plus pratique et un diplôme de «master» dès 1945, mais les autorités éducatives mettront du temps à reconnaître l’institution. Dans les années 1950, l'université de São Paulo bénéficiera d'une aura plus prestigieuse et d'un financement plus régulier et remportera la compétition. Dans les années 1940-1950 les étudiants circulent cependant régulièrement entre les deux institutions, faisant par exemple leur «master» à l'ELSP et le doctorat à la USP ${ }^{\mathrm{1} 2}$.

\section{NOGUEIRA ET LE MONDE ACADÉMIQUE BRÉSILIEN}

Nogueira naît à Cunha, bourgade rurale de l'état de São Paulo dans une famille d'instituteurs, catholiques fervents ${ }^{13}$. Soldat à 15 ans lors de la révolte pauliste de 1932, puis journaliste dans une feuille locale et militant au parti communiste (Cavalcanti, 1999), il est très jeune attaché à défendre la «justice sociale» (Nogueira, 1995). Cet activisme précoce est cependant ralenti entre I936 et 1938 lorsqu'il soigne sa tuberculose dans un sanatorium. À sa sortie, il suit ses parents dans la carrière d'instituteur. Le virage vers la sociologie se fera en 1940 après la réussite au concours de l'École Libre de Sociologie et de Politique qui proposait des bourses de poursuite d'études. Nogueira suit le cursus de l'école jusqu'en 1945, année où il obtient le mestrado (master) avec un mémoire s’appuyant sur son expérience en sanatorium (Nogueira, 1950).

II Par exemple: Willems (1940) sur les populations immigrés, Willems (1947) sur le village de Cunha, Pierson (1945) sur les contacts raciaux.

12 Voir le témoignage de Juarez Brandão Lopez dans Valladares (2006).

13 Sur la biographie de Nogueira: Cavalcanti (1995, 1996). 
Dès 1942, il commence à enseigner dans la propre institution où il étudie. De I945 à 1947, il est à Chicago grâce à une bourse de l'Institute for international education et suit des cours de sociologie et d'anthropologie. Il revient en 1947 pour effectuer son travail de terrain pour le doctorat, mais, en 1952, les autorités américaines lui refusent le visa pour activisme communiste. La recherche est pourtant effectivement entamée : une étude de communauté dans la bourgade d’Itapetininga dans l'état de São Paulo. Cette enquête qu'il va mener de façon intermittente alimentera l'essentiel de ses écrits des années 1950 en fonction des thèmes choisis ${ }^{14}$. Sans situation institutionnelle solide il vit alors, comme d'autres chercheurs de son époque, de petits contrats et de cours donnés notamment à l'ELSP jusqu'au départ de Pierson au milieu des années 1950. Il officie également à l'Institut d’administration de la faculté d'économie de São Paulo d’abord comme technicien remplaçant puis en gravissant les échelons. Entre 1957 et 196I, il est est au Centre brésilien de recherches éducationnelles (CBPE) de Rio de Janeiro, dirigé par Darcy Ribeiro ${ }^{15}$ lui aussi ancien élève de Pierson. Dans les années 1960, il enseigne dans diverses petites universités et cherche des contrats pour faire vivre sa famille. Les portes de la prestigieuse faculté des lettres de la USP lui seront en fait fermées jusqu’en 1970, date à laquelle Florestan Fernandes, adversaire acharné de Pierson, est en exil. Il ne sera finalement admis au dernier rang universitaire de professor titular qu'à la faculté d'économie en 1978 (Nogueira, 1995).

Tout au long de cette carrière en demi-teinte, Nogueira restera, tout au moins d'un point de vue méthodologique, très proche de Pierson. À l'ELSP, malgré le grand nombre de gens doués à l'époque, c'est Nogueira qui est le disciple principal du maître américain et qui l’assiste notamment pour les

14 Le terrain commencé en 1947 (Nogueira (1962) donne lieu ainsi à un premier compte rendu sur les relations raciales. Entre 1952 et 1953, l'enquête est poursuivie par un recueil d'informations sur les paysans dans le cadre d'un contrat avec le ministère du travail puis en 1956, le terrain est repris pour une enquête sur l'éducation.

15 Né en 1922 dans l'état du Minas Gerais il a étudié à São Paulo et s'est spécialisé en anthropologie. II a ensuite travaillé pour le Centre de protection des Indiens (1947-1956) et plus tard au Centre brésilien de recherches éducationnelles à Rio de Janeiro. II a ensuite contribué à fonder l'université de Brasilia qu'il dirigea et a, par la suite, occupé des positions de premier plan au sein du dernier gouvernement démocratique des années 1960. 
enseignements d'initiation à l'enquête. Certains étudiants étaient enthousiasmés par la forme concrète de sociologie enseignée à l'ELSP car elle proposait la perspective d'une étude «scientifique » des problèmes brésiliens ${ }^{16}$. Le mémoire de mestrado de Nogueira publié en 1950 suit cette orientation empiriste et se fonde sur ses souvenirs mais aussi de l'observation, des entretiens, et l'analyse de documents personnels (Nogueira, 1995).

\section{LA SOCIOLOGIE DES RELATIONS RACIALES AU BRÉSIL VERS 1940}

À la fin des années 1930, la sociologie des «relations raciales » n’existe pas en tant que telle mais trois lignes dominent la réflexion sur la place des Noirs dans la société brésilienne. La première peut-être rattachée à Oliveira Viana. Juriste de formation et disciple de Silvio Romero ${ }^{17}$, Viana est au début des années 1920 l'un des principaux spécialistes de la formation du peuple brésilien. Mais, adepte d'une vision biologique de l'étude des races, il est très fortement critiqué dans les années $1930^{18}$. Son anthropologie se veut un produit des avancées de la biologie des races qui montrerait que les groupes raciaux sont inégaux dans la mesure où ils ne produisent pas dans les mêmes proportions des individus intelligents et des tempéraments adaptés à la civilisation (Viana, 1932). Les théories de Viana sont donc des théories racistes qui resteront jusque dans les années 1940 insensibles aux avancées de l’anthropologie culturelle (Ramos, 2003).

16 Darcy Ribeiro, alors étudiant avec Pierson écrit par exemple à Nogueira (10/28/1943) une lettre dans laquelle il appelle de ses vœux l'«établissement au Brésil des disciplines de sciences sociales comme de véritables sciences» (Archives Nogueira à l'Institut de philosophie et de sciences sociales (IFICS) de l'université fédérale de Rio de Janeiro- désormais: AN-IFICS).

17 Silvio Romero fut le plus célèbre critique littéraire de la fin du dix-neuvième siècle et a exercé une influence considérable sur de nombreux intellectuels du début du siècle suivant; voir Candido (1987).

I8 On se reportera notamment aux critiques caustiques faites par Emilio Willems (1955, 1957). De même il n'était pas pris au sérieux par les Américains comme Pierson ou comme Frazier qui note de façon ironique dans son compte rendu en 1936: «lt is interesting to note in passing that such pronouncement concerning the inferiority o the negro by a mulatto in a country where race does not determine social status assume a purely academic character» (Frazier, 1936, p. 675). 
La deuxième concerne les études anthropologiques conduites dans la lignée de Raimundo Nina Rodrigues (I862-1906) qui s’intéressent moins aux «relations raciales » quaux Noirs en eux-mêmes. Spécialisé en médecine légale et disciple de l'école italienne d'anthropologie criminelle ${ }^{19}$, Nina Rodrigues s'occupait en particulier de la mise en évidence de l'héritage traditionnel africain et des particularismes des Noirs pauvres de Salvador. Ses enquêtes le conduisent à la conclusion que la race noire est attardée, infantile et doit être soumise à un code pénal adapté. Arthur Ramos (1903-1949), l'un de ses disciples joue un rôle encore plus important dans la discipline. Fils d’un médecin d’Alagoas, il étudie la médecine légale à Salvador puis enseigne la psychologie et l'anthropologie à l'université du District Fédéral à Rio dans les années 1930. Il s’intéresse comme Nina Rodrigues aux problèmes d'inadaptation des Noirs à la société brésilienne d’abord d'un point de vue psychiatrique puis culturel: s'il nie les idées racistes, il pense que la culture noire est en retard et freine l'intégration (Ramos, 1934). Dans les années I940 cependant, il suivra ensuite l'enseignement d'Herskovits à Northewestern et adoptera une position plus nettement culturaliste et antiraciste (Ramos, I943) lui permettant d'être pleinement reconnu et de travailler pour l'Unesco.

La troisième influence déterminante vient de Gilberto Freyre. Bien qu'il n'étudie pas les relations sociales de son temps, Freyre, formé à l'anthropologie à l'école Boasienne, décrit dans ses premiers livres, entre autres choses, les liens entre Blancs et Noirs de l'époque coloniale jusqu'à la République. Son livre phare Casa grande e senzala (1933), montre l'importance de l'héritage nègre dans la culture et la vie sociale brésiliennes (Chacon, 1993). Pour ce qui concerne les relations raciales, il montre que le métissage a dès les origines miné la rigueur de l'ordre patriarcal qui aurait dû, s'il l'avait pu, séparer strictement les races et les classes, comme dans d'autres sociétés esclavagistes. La pensée de Freyre a cependant été régulièrement déformée. On a ainsi souvent retenu l'idée qu'il affirmait que la société brésilienne était dépourvue de racisme ${ }^{20}$. Comme l’a

19 Au sujet de Nina Rodrigues et de sa postérité, voir Mariza Correa (2000).

20 L'interprétation déformante réduisant l'œuvre de Freyre à une défense des excès des Blancs lors de la période coloniale est toujours vivace; voir par exemple: Nascimento et Nascimento, 2001. L'«école de São Paulo» dans les années 1950-1970 a également relégué les 
bien montré Lévy Cruz (2003), cette manière de présenter les choses est une déformation des résultats des travaux de Freyre mais elle constitue un pôle des débats (politisés) sur le sujet.

Il n'en demeure pas moins que les travaux de Freyre ont été lus avec passion par les étudiants de São Paulo. Du côté de la USP, Bastide a traduit Casa grande e senzala tout en se montrant critique vis-à-vis de la question du racisme et de la douceur lusophone des rapports raciaux. Le chemin emprunté par Bastide est, quant à lui, tout entier orienté vers la défense de la culture afro-brésilienne. Dès 1938, il avait entrepris de publier des textes attirant l'attention sur la position reléguée des Noirs ${ }^{21}$. Bien qu'il ne pu entreprendre des recherches empiriques dans le Nordeste qu'en 1944, il rejoignait la perspective d'Herskovits, voyant dans les populations noires des héritiers du monde africain dont la richesse culturelle devait être étudiée et défendue.

Du côté de l'ELSP, Pierson apporte l'idée d’une sociologie des relations raciales, radicalement différente de l'étude des groupes pour eux-mêmes. Dès son arrivée en 1939, il publie des textes sur la base de sa thèse ${ }^{22}$. Sa perspective est celle de Park qui se propose d'étudier à l'échelle mondiale les conséquences relationnelles de l'expansion européenne (Chapoulie, 2002). La première question qu'il pose est de déterminer s'il y a bien présence de groupes ethniques ou raciaux au sens sociologique du mot groupe. Après deux années de travail à Salvador de Bahia, Pierson conclut qu'il n'y a pas de groupe noir autonome, que la culture africaine est en déclin et ne fournit pas l'armature d'un groupe social distinct, qu'il n'y a pas de ségrégation spatiale et que les relations les plus importantes sont des relations de classe et non de race. On ne peut donc pratiquement pas parler de relations raciales. Cela ne signifie pas en revanche que la couleur d'un individu n'ait pas une certaine importance dans le déroulement de son existence, mais cette importance sera toujours moindre que celle due à

idées de Freyre à une position secondaire (Velho, 2008).

21 Voir, par exemple, Bastide (1939, 1943, 1950). Notons que le Français Pierre Denis (1909) avait déjà souligné le problème de la position des Noirs.

22 Son livre tiré de sa thèse en anglais, Negroes in Brazil est publié en 1942 puis traduit en portugais en 1945. 
d'autres caractéristiques sociales. Il est décisif de le souligner que, dans ce cadre, Pierson n'étudie pas le racisme, pas plus qu'il n'étudie les relations sociales à partir des perspectives et des intérêts de l'un des groupes. Il ne cherche pas à savoir si la société brésilienne est à blâmer ou à féliciter mais quelle est sa manière d'organiser les conséquences des successives rencontres raciales. Le projet de Pierson se heurtait ainsi aux habitudes intellectuelles brésiliennes puisqu'il ne visait pas à faire un diagnostic de l'état du peuple brésilien et ne tentait pas de dénoncer une injustice.

\section{PREMIÈRES EXPLORATIONS DES RELATIONS RACIALES}

Au début des années 1940, Nogueira se trouve plongé dans ce monde de débats tranchés, et occupe de ce fait une position particulièrement intéressante pour nous. Il connaît la perspective américaine ainsi que la rigueur scientifique de Pierson. Mais en même temps, il veut aider à améliorer le monde. Pour autant il n'est pas lié aux mouvements noirs comme Bastide et il n'est pas intéressé par le folklore africaniste. Ce n’est pas non plus un idéologue ni un adepte du marxisme. La voie que va suivre Nogueira est en fait assez unique : il va essayer de trouver une interprétation de la situation raciale brésilienne combinant une sensibilité envers le racisme, un intérêt pour les «race relations» et une croyance en l'enquête empirique.

Pour ce faire, il prend son inspiration dans les travaux de Pierson mais avec des réserves. Mettre au clair ce que Nogueira retient exactement de Pierson demande une lecture attentive des travaux des deux hommes. D'un point de vue méthodologique, l'élève est très attaché à l'héritage empiriste du maître jusqu'à la fin de sa carrière (Nogueira, 1964). Mais en matière de «relations raciales », il est assez évident que Nogueira s'écarte des idées de Pierson sur au moins trois points :

1. le racisme envers les Noirs doit être étudié et fait toujours partie de la «situation raciale» brésilienne;

2. la question de la situation raciale peut être traitée en partant de la subjectivité des gens en position d'infériorité; 
3. mettre à jour un point de vue négatif généralisé sur les Noirs suffit à prouver que la vie sociale et la mobilité sociale sont affectés par les «préjugés raciaux».

Sur ces questions, Pierson a une opinion opposée. Selon lui, le terme de «racisme» est flou dans le cas brésilien et n'a qu'une importance secondaire vis-à-vis de la question de l'existence éventuelle de groupes raciaux. L'étude des «relations raciales » se fait selon lui en examinant le fonctionnement de la société entière dans le temps. Elle ne peut se limiter à mettre en évidence des «problèmes raciaux » constitués par les plaintes d'un segment de cette société. Par ailleurs, fidèle aux indications de Blumer, Pierson considère qu'il y a une différence radicale entre préjugé culturel négatif et discrimination effective, notamment pour ce qui touche à la mobilité sociale.

Ces divergences sont évidentes à la lecture du premier texte de Nogueira sur le sujet. En 194I, le jeune sociologue profite de l’absence temporaire de Pierson pour conduire une rapide enquête sur le racisme à l'embauche (Cavalvanti, I999) car il était insatisfait de la manière dont son professeur avait traité le thème. Pour comprendre ce point il faut rappeler ce que sont exactement les idées de Pierson au sujet du racisme. Ses détracteurs lui feront dire par la suite qu'il a nié l'existence du racisme et de la discrimination au Brésil²3. Pierson serait donc l'un des maillons d'un courant de pensée qui considère qu'il n'y a pas de «race problem» au Brésil ${ }^{24}$. Mais tout dépend en premier lieu de la façon dont les termes sont définis. Quand Pierson dit qu'il n'y a pas de «race problem» il entend par là qu'il n’existe pas de groupes raciaux constitués en situation de conflit. Le «problème» évoqué est un problème pour la société, pas pour les gens qui se plaignent de leur existence. Quand Nogueira, Bastide, Willems et plus tard Fernandes parlent d'un «problème racial», ils veulent dire qu'une partie de la population souffre de ce qu'elle ressent comme des injustices ou des humiliations. Pierson s'est en fait expliqué très clairement (Pierson, I95I) : s'il ignore le «racisme» dans son livre, c'est qu'il n'y voit pas l'élément moteur de l'organisation sociale brésilienne. Le fait que des gens de couleur diffé- 
rentes ne s'apprécient pas lui semble une banalité, courante partout dans le monde. Il existe donc bien au Brésil des préjugés de couleur, de classe et de culture comme partout ailleurs (Pierson, 1951). Mais ce qui est notable, selon lui, c’est que le Brésil est une société compétitive dans laquelle la réussite individuelle ne dépend que secondairement de la race ou de la couleur ${ }^{25}$. Les mécanismes à l'oeuvre ressemblent à ceux existant au sein de groupe noir américain (Pierson, 1945) : la noirceur de la peau est un handicap mais ce sont les classes sociales qui sont le facteur déterminant.

Nogueira a sans aucun doute compris ce point de vue dès le début des années I940, ce qui ne signifie pas qu'il l’apprécie ni l’accepte. Adepte d’une sociologie à la fois rigoureuse et militante, il va prendre un autre objet, moins global, et plus lié aux sentiments individuels. Frappé par l’abondance d’annonces de journaux spécifiant que seules des domestiques blanches sont demandées, il se met en tête de vérifier l'importance éventuelle du racisme (Cavalcanti, 1999). Aidé d’autres étudiants, il entreprend d'interviewer une partie des employeurs pour en connaître les raisons (Nogueira, 1942). Sur 223 interviewés, seuls 2 refusent de répondre et les autres donnent une série de justifications allant de propos ouvertement racistes à l'incapacité d'expliquer leurs actes. Nogueira ne classe pas vraiment les réponses et ne cherche pas à les intégrer dans un schéma global d’analyse: il lui semble suffisant d’avoir montré que des mécanismes racistes sont à l'œuvre (Nogueira, 1995, p. 125).

Le texte est bien accueilli par les défenseurs de la cause noire ${ }^{26}$ et par les sociologues qui n’appréciaient pas les conclusions de Pierson. Selon Willems, par exemple, l'article montrerait de façon claire l'existence d'un préjugé qui ne peut être confondu avec le préjugé de classe (Willems, 1949, p. 404).

25 Voir en particulier «A situação racial brasileira» conférence prononcée en 1940, et republiée en 1945 dans l'ouvrage de synthèse: Teoria e pesquisa em sociologia.

26 Notamment par Fernando Goes (journaliste métis originaire de Bahia) et Mauricio de Medeiros (médecin métis, écrivain et académicien); voir à ce sujet Nogueira (1995). 
Le point reste cependant à démontrer car, bien qu'il fût au cœur du sujet, Nogueira n'a pas cherché à l'étayer. Sachant que les groupes de population les plus pauvres, ceux dont l'éducation est la plus limitée et les manières les plus frustes, étaient constitués de Noirs non métissés, il est tout à fait possible, comme le pensait Pierson, que le mépris «racial» visait en fait la figure du sous-prolétaire ou de l'immigré rural. Si l'on examine les réponses citées dans l'article, on voit en effet que les déclarations ouvertement racistes (par exemple «les Noirs sentent mauvais ») sont peu nombreuses (à peu près 25 sur le total). En revanche, beaucoup de répondants (48) ne savent pas expliquer leur préférence pour des employées blanches, 27 nient avoir une préférence, i 2 le font par habitude des employées blanches, une vingtaine, notamment des professionnels, déclarent que les employées blanches font meilleure impression auprès de la clientèle. Par ailleurs, un grand nombre de réponses (à peu près 70) peuvent fort bien correspondre à un rejet des gens assimilés aux basses couches de la population. Certains employeurs disent refuser les Noirs pour leur manque de responsabilité, de ponctualité, de discipline ou pour leurs manières grossières : défauts habituellement reprochés aux sous-prolétaires. Nogueira ne fait pas l'effort de départager le rejet d'un type social (sous prolétaire ou paysan) avec celui de la personne de couleur. En outre, une partie des répondants finissent par embaucher des Noirs ou des Métisses ou en avaient déjà à leur service. Si l'article montre donc bien que les gens de couleur sont associés aux segments les plus frustes de la société, il échoue à discuter l'hypothèse la prééminence du préjugé de classe. La conclusion de Nogueira laisse entendre que la question reste ouverte. Pour trancher, il aurait fallu enquêter sur l'embauche d'individus de classe moyenne et donc aller au-delà de la simple dénonciation du racisme.

\section{UN SOCIOLOGUE BRÉSILIEN À CHICAGO}

Nogueira commence à suivre les cours à l'université de Chicago en octobre 1945. Il apprécie certains cours mais se montre, comme d’autres étudiants brésiliens avant et après lui, un peu déçu par les enseignements undergraduate. 
Au premier semestre 1945, il suit le cours «Comparative institutions» par L. Warner qu'il juge bâclé, «Statistiques» avec Burgess qu'il juge mauvais et «Anthropologie» avec Cole qu'il adore ${ }^{27}$. Au cours du deuxième trimestre, il suit deux cours de Hughes qu'il apprécie («Introduction to field studies» et «Cultural and racial contacts») $)^{28}$ et un cours de Wirth («Social organization»), qu'il encense ${ }^{29}$. La participation à ces cours l'oblige à un travail intense auquel il n'était pas habitué au Brésil ${ }^{30}$. Au début de son séjour, il se montre globalement déçu par le système américain : les professeurs sont, selon lui, sur-spécialisés avec chacun sa théorie, les examens sont corrigés par des chargés de cours, le niveau général des étudiants undergraduates est «bas», les professeurs enseignent une «répétition déformée des grands maîtres européens $»^{31}$.

Parallèlement, il commence à recueillir des données sur les contacts raciaux locaux. Il entre en contact avec Horace Cayton dès décembre 1945 et participe aux réunions de divers organismes de lutte contre la discrimination $(\text { dont le NAACP })^{32}$. Le milieu étudiant qu'il découvre à Chicago est globalement

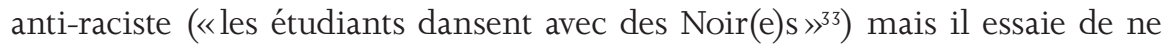
pas s'y enfermer. Il visite le quartier noir et prend des notes. Il est alors fasciné par la définition américaine de l'appartenance au groupe noir : de nombreuses personnes considérées comme blanches au Brésil ne le sont plus à Chicago, telle cette femme blonde, en fait une Noire, rencontrée dans une réunion (Nogueira, 1995). Il perçoit alors clairement à quel point les classifications sont souples et imprécises au Brésil. Ainsi, lorsque Cayton lui demande s'il est blanc ou «moreno», il ne sait pas répondre ${ }^{34}$.

27 On se reportera au dossier 9 des archives Pierson au Centre Edgar Leuenroth à l'UNICAMP (désormais: AP-CEL).

28 Lettre à Pierson du 29/01/1946 (AP-CEL).

29 Voir lettre de Nogueira à Cyro Berlinck (directeur à l'époque de l'ELSP) du 29/0I/46, AN-IFICS.

30 Lettre à Pierson du 25/03/1946, AP-CEL.

31 Lettre à Rodolfo du 06/09/1946, AN-IFICS.

32 Voir le récit résumé après coup de ces expériences dans Nogueira (1995).

33 Lettre à Pierson du 25/03/46, AP-CEL.

34 Lettre à A. R. Muller du 10/10/1946, AN-IFICS, dossier 2. 
Son séjour aux États-Unis est donc un moyen de réfléchir à la situation brésilienne, notamment à travers la vision qu'ont les Américains de cette situation. Dans un travail rendu à Hughes en 1946, Nogueira affirme ainsi que «Les Américains voient les Latino-Américains à l'aide d'une grille raciale qui est inconsistante avec l'idéologie et la situation raciale de la plupart des pays latino-américains. L’Américain blanc tend à suggérer au Latino-Américain blanc son propre schéma d’attitudes raciales et le Noir américain tend à exagérer les faits concernant la rela-

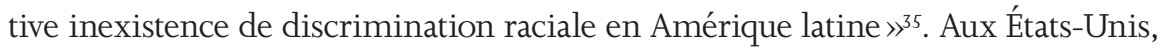
les Brésiliens à la peau brune sont victimes de discrimination au même titre que les Noirs, ce qui attise chez eux une «race consciousness » inexistante chez des gens qui sont habitués à un ordre social qui méprise le racisme. En 1947, Nogueira adresse sur la base de ces réflexions des critiques sévères (Nogueira, 1947) à l'historien Tannenbaum dont le livre (Slave and citizens: The Negro in the Americas) montre selon lui que les auteurs américains ne connaissaient pas en profondeur la société brésilienne. Ils ne perçoivent pas la diversité des tons et des catégories de couleur, ils oublient qu'il n'existe pas véritablement de «color line» au Brésil et que la solidarité raciale n'existe pratiquement pas.

Le point qui gêne le plus notre auteur est qu'il hésite entre une posture militante et l'exigence de neutralité affirmée à Chicago : «Peut-être est-ce mon éducation catholique qui fait que j’ai encore une terrible résistance à accepter par exemple l'idée que le lynchage soit une chose “naturelle”, que le préjugé racial ait une fonction sociale, celle de maintenir la structure de caste de la société dans laquelle il se manifeste, et ainsi de suite $»^{36}$. Ces hésitations sont l'occasion pour Nogueira de mûrir les critiques qu'il adresse petit à petit à la sociologie. Pour lui, non seulement cette dernière doit s'occuper des «burning questions », mais encore, elle doit le faire de façon engagée. Ainsi dans le term paper qu'il rédige pour le cours de J. D. Lohman au printemps 1947, écrit-il: «C'est parfois mon sentiment que les sciences sociales aux U.S.A. sont trop

35 «Outline of a field study: the meaning of Latin America for american negroes», term paper adressé à Hughes dans le cadre de son cours «Advanced field studies», automne 1946.

36 Document non daté mais probablement de 1946, AN-IFICS, dossier 2. 
imprégnées d'une attitude "neutre" ou de "laissez-faire" ${ }^{37}$. De son point de vue, même Myrdal est trop neutre: «Je pense que le rôle du sociologue est d’aider les déshérités et pas de fournir une justification de plus à ceux qui souhaitent maintenir le modus vivendi actuel $»^{38}$. Dès lors, selon lui, le sociologue devrait éviter toute publication donnant des armes aux conservateurs. De même, enseigner que la recherche est pratiquement une fin en soi et que le jugement doit s'y suspendre temporairement lui semble une bizarrerie: «Il y a peu, dans un cours, un étudiant a demandé au professeur s'il était vrai qu'en Amérique latine, les étudiants font des révolutions parce qu'ils ne jouent pas au football ${ }^{39}$. Et le professeur a répondu: “nous n'avons pas encore assez de recherche sur cette théorie”.» Nogueira, qui ignore sans doute les débats anciens à Chicago entre sociologues et militants sociaux (Chapoulie, 200I), est consterné par cette attitude qu'il croit couramment admise.

Pourtant sa manière d'envisager concrètement les études raciales est moins militante qu'elle ne paraît. Ainsi dans la une première mise en forme de ses idées, adressée à Lohman, le Brésilien se déclare-t-il globalement en accord avec Freyre bien que ce dernier « devrait prêter plus d’attention aux cas négatifs qu'il ne le fait d'habitude $\gg^{4^{\circ}}$. Il conçoit également, comme Pierson, que le sujet impose une étude des relations entre groupes sur la base de ce que l'on sait des relations sociales au sein de chaque groupe. Dans ce cadre, la race ne peut être que l'un des facteurs réglant les relations sociales : «La race comme facteur de statut devrait être étudiée en même temps que d’autres facteurs de statut et par comparaison avec eux au sein de la même structure sociale. $\gg^{41}$. Il ne s'agit donc pas de regarder les Noirs seulement comme des Noirs. Autre point non-conformiste tiré d’observations de terrain : beaucoup de Noirs américains manifestent une «hyper conscience» raciale «attribuant au préjugé racial toutes les

37 «Case study in racial and cultural tension », AN-IFICS, dossier 2.

38 Ibid.

39 Lettre à Rodolfo, 24//1//946, AN-IFICS.

40 «Case study in racial and cultural tension », op.cit.

4 I Ibid. 
attitudes décevantes venant des autres $\gg^{42}$. Les tensions entre Noirs et Blancs s'expliqueraient ainsi au moins en partie par les anticipations de racisme de la part des gens de couleur: «Combien l'attitude et le comportement du Noir américain sont conditionnées par ces attentes». Cette conviction est notamment renforcée chez Nogueira par les relations qu'il a entretenues pendant plusieurs mois avec un Noir de petite classe moyenne à qui il donnait des cours de portugais ${ }^{43}$. L'homme en question, selon les critères de notre auteur, se révéla rapidement «racialy over conscious » dans la mesure où il attribuait toutes les limitations de son existence au racisme. Ce cas inspira des réflexions sévères à Nogueira : «Je ne peux manquer de réfléchir sur cet égocentrisme qui rend le Noir américain aveugle en relation à n'importe quel autre problème qui ne soit pas le sien ainsi que sur cette lâcheté avec laquelle il fuit la lutte dans laquelle il est la part la plus intéressée. »

Ce type de considérations lui permit en tout cas de regarder avec plus de recul la situation brésilienne : bien que plus favorable aux Noirs, elle n’empêche pas l'existence d'un «certain colour snobishness in Brazil» selon le mot employé dans son texte pour Lohman. Le terme est beaucoup plus atténué que celui de racisme utilisé habituellement au Brésil et surtout, il insiste sur la couleur. Nogueira voit la similitude avec le mépris pour les Noirs foncés décrit par Cayton et Drake (1945) au sein même du groupe noir. Ce snobisme de couleur n'est cependant pas totalement séparé par notre sociologue de la situation de classe. Bien qu'il ne théorise pas la relation entre les deux, Nogueira propose l'hypothèse que le Brésil est surtout une société de classes et que si les clivages de classes ne satténuent pas ils pourraient mener à un conflit de classe ou de race déguisé. L’absence de conflit racial viendrait d’une société ou chacun a sa position et l'accepte ${ }^{44}$. Dans les petites communautés où tout le monde se connaît, la couleur gêne peu. En revanche dans les grandes villes, les gens de couleurs sont victimes de certaines «erreurs» (des confusions de position

42 Ibid.

43 Les données exploitées dans ce texte viennent de notes de terrain très complètes prises par Nogueira au jour le jour en 1946 et 1947.

44 Lettre à Pierson du 27/II/1946, AP-CEL. 
de classe en fait), accumulées, peuvent «attiser leur conscience de race ou de couleur $\gg^{45}$. Par exemple un médecin noir est assis dans sa voiture et un homme le prenant pour un chauffeur de taxi s'assoie sur le siège arrière. De même, une vieille dame noire est prise pour une domestique à l'entrée d'une organisation religieuse. Ainsi Nogueira, malgré son militantisme, aboutit, sur la base de ce qu'il observe, à des idées très proches de celles de son ancien professeur, lui-même considéré comme un conservateur au Brésil. La couleur noire est, dans cette optique, essentiellement un indice de la position sociale et les humiliations vécues par les Noirs viennent souvent du mépris de classe.

Ce n'est cependant pas entièrement sur la question des relations raciales que Nogueira envisage de travailler de retour au Brésil, puisqu'il fait approuver en août 1947 un projet de thèse en forme d'étude de communauté («The status system of a brazilian town ») ${ }^{46}$. Hughes, qui est celui qui a le plus suivi le dossier n'a apparemment aucun doute sur la réussite future de Nogueira dont l'une des qualités serait justement la capacité à faire du travail scientifique plutôt que du militantisme politique ${ }^{47}$.

\section{NOGUEIRA ET LE PROJET UNESCO}

Après que sa demande de visa lui a été refusée, Nogueira poursuit son travail de terrain à Itapetininga et va utiliser ses données pour une enquête de grande visibilité. Comme on le sait (Chor maio, 1997) l'Unesco au début des années 1950 lance au Brésil une série de recherches sur les relations raciales afin d'explorer les secrets d'un modèle de société multi-raciale pacifique. Donald Pierson engagé dans ses travaux sur les bourgades du Nordeste, décline l'offre mais indique son élève Nogueira (Chor Maio, 2005). Nogueira est conscient dès le départ que les recherches qui seront chapeautés à São Paulo par

45 Term paper pour le cours de Lohman.

46 Lettre à Pierson 27/08/1947 (AP-CEL).

47 D'après Pierson (lettre à Nogueira du 28/04/1952, AN-IFICS) Hughes avait déclaré: «I never knew anyone who devoted himself so thoroughly to his main business which was not politics ». 
Bastide ont très clairement une intention militante, ce qui ne le dérange pas ${ }^{48}$. Bastide dès ses négociations avec Métraux qui coordonne l'opération a annoncé que les buts étaient de faire changer les points de vue sur le racisme, de favoriser la coopération entre intellectuels blancs et associations noires et d'influencer les actions gouvernementales (Chor Maio, 2005). Nogueira ne se départit cependant pas de la prudence et de la rigueur apprises à l'ELSP. Sur la base de ses travaux en cours, il propose en effet un projet de recherche qui, à l'image de celui proposé par Pierson lors de sa thèse, laisse les questions ouvertes ${ }^{49}$.

Ainsi, il fait valoir les étapes suivantes:

1. conclusions à prévoir : constatation de l'existence ou de l'inexistence de préjugé, antipathie, ségrégation, dissensions, ou conflit entre les divers composants ethniques de la population;

2. constatation de la tendance des modèles de relations interraciales à changer dans la direction de modèles plus ou moins libéraux;

3. constatation du changement ou non dans les manières par lesquelles les individus porteurs de traits raciaux jugés indésirables réagissent au préjugé ;

4. constatation du changement ou non de l'attitude de la part d'éléments du groupe blanc vis-à-vis des qualités d'éléments noirs ou négroïdes.

Les résultats du travail de Nogueira sont publiés en partie dans la revue Anhembi et en partie à la fin du livre de Fernandes et Bastide (1955) sans que le lecteur ne sache exactement le lien entre son enquête et celle des auteurs du livre ${ }^{5}$. Nogueira s'en est d'ailleurs plaint en ces termes à Paulo Duarte, rédacteur en chef de Anhembi : «Mon travail ne peut être considéré ni comme une partie subsidiaire ni comme un appendice du travail réalisé par nos amis communs, les illustres professeurs Bastide et Fernandes, pas plus qu'il ne peut

Voir la lettre adressée Orlando Barbosa le 25/I0/1950 (AN-IFICS).

49 Ce ne sera pas le cas pour d'autres volets de la recherche de I'UNESCO, notamment ceux proposés par Costa Pinto et Bastide et Fernandes dans lesquels les réponses sont posées avant la recherche.

50 Cavalcanti (1999) signale que le sous titre du livre concernait São Paulo et excluait donc le travail de Nogueira. Par ailleurs seul l'un des deux textes prévus fut finalement publié. Enfin, la deuxième édition du livre en 1959 exclut les contributions de Nogueira. 
être présenté comme ayant été exécuté sous l'orientation de ces chercheurs, comme cela est fait sur la couverture du volume..$^{{ }^{11}} \gg$ Le spécialiste d'Itapetininga voulait donc une publication indépendante que l'on ne lui accorda pas.

La lecture du plus connu des textes issus de cette recherche (Nogueira, 1955) montre que les désaccords vont au-delà d'une simple question de publication. Cet article, encore régulièrement cité aujourd'hui (dont le titre en français serait : «Préjugé racial de marque et préjugé racial d’origine») est destiné à comparer le préjugé existant au Brésil et celui en vigueur aux États-Unis. Le texte s'oppose à Pierson qui n'est pas nommé mais bien visé quand il s'agit de critiquer ceux qui se «refusent à accepter que le problème du préjugé racial soit le problème central dans les études de relations raciales» (p. 279). Mais il s'oppose aussi à Costa Pinto en affirmant que les préjugés raciaux étudiés sont différents en nature et pas seulement en intensité.

Dans la suite, Nogueira se sert de ce qu'il a appris aux États-Unis et décrit finement le préjugé brésilien à partir de ses observations. Il en ressort, conformément à ce qu'avait vu Pierson et contrairement à ceux qui dressent un portrait dramatique de la situation: que le préjugé varie selon l'intensité de la couleur dans une société où la ligne de couleur est flottante et les groupes raciaux inexistants en tant que tels, qu'il n'empêche pas les relations d'amitié et une vie normale au milieu des Blancs, qu'il ne manifeste ses effets que dans des cas rares (en principe des disputes) dans une société qui méprise le racisme. Nogueira décrit en fait la couleur noire comme une sorte de défaut social qui peut être compensé par d'autres qualités et n’est gênante que face à des étrangers dans une grande ville. Il n’en conclut pas moins que la «probabilité d'ascension sociale est en raison inverse » de l'intensité de la couleur. C'est-à-dire qu'il juxtapose des éléments d’analyse avec lesquels Pierson était tout à fait d’accord avec une conclusion qui va dans le sens de Bastide et Fernandes. Le passage de la première partie de son analyse, préjugé esthétique peu dévalorisant, à la seconde, mobilité sociale bloquée, n’est pas étayé par une démonstration. C'est là une caractéristique de toute l'œuvre de notre auteur au sujet des 
races au Brésil: ses observations de la vie sociale ne corroborent pas complètement son analyse de l'évolution de la structure sociale. On le constate mieux dans la partie centrale de son travail d'étude de communauté.

\section{ÉTUDE DE COMMUNAUTÉ ET RELATIONS RACIALES}

L'enquête de Nogueira, faite en dehors des grandes universités et fidèle à un modèle d'étude passé de mode dans les années 1960 a été à peu près oubliée jusquaux années 1990. Pourtant elle constitue une contribution décisive à l'étude des «relations raciales». Ce texte dense (Nogueira, 1998) étudie la nature et l'évolution des relations sociales et raciales dans une bourgade en tenant compte de la structure sociale et de l'économie locale. Comme Pierson avant lui, Nogueira a travaillé lentement en recueillant choses vues et entendues à partir de la position d'un homme installé dans la communauté. Pierson cependant cherchait à répondre à des questions précises sur les éventuels groupes raciaux. Nogueira, lui, a recueilli des informations variées et a accordé beaucoup d'importance à la reconstitution historique. Dans le contexte des débats que l'on a mentionné, ce texte est finalement un document tout à fait étonnant. En effet, notre auteur reste dans l'ensemble fidèle aux méthodes d'enquête de Pierson, à une partie de son vocabulaire et aux outils théoriques de Chicago. En même temps, son texte vise clairement à démontrer qu'une certaine forme de racisme joue sur le long terme un effet de ralentissement de la mobilité sociale des gens de couleur.

Si l'on parcourt rapidement le texte, les propos de Nogueira, étayés par de nombreuses données empiriques, sont très convaincants. Si l'on examine en détail les modes d’administration de la preuve, on constate en revanche qu'à défaut de véritablement prouver l'existence d'un racisme agissant sur les carrières, il parvient surtout à confirmer que le statut social de la négritude est bas. Dans le quatrième chapitre en particulier il montre avec un grand luxe de détails que le fait d'être noir est associé à un statut déprécié, ce qui n’est guère étonnant à la campagne 60 ans après la fin de l'esclavage. Les proverbes le rappellent comme les déclarations des enfants ou les interactions du quotidien: être 
noir n'est pas valorisé et l'on évite de parler ouvertement de la couleur foncée d'une personne. Mais une lecture attentive montre que Nogueira ne parvient pas à prouver que ce fait entraîne une relégation des Noirs supérieure à celle envisagée par Pierson. En effet, son argument principal est que les Noirs citent facilement des «exemples (...) qui montrent comment la société nationale réduit la mobilité sociale des Noirs et des Métis et leur réserve des humiliations épargnées aux Blancs dans des conditions égales (p. 196) ». Autrement dit, au moment de prouver sa conclusion principale, Nogueira confie le rôle de sociologue aux répondants. De même, l'évitement des Noirs par les Blancs est documentée par des déclarations au sujet de ce qui est susceptible de se passer. Quand il s'agit de montrer l'avantage des Blancs en compétition avec les Noirs, l’auteur n’est plus capable de citer des notes d'observation. Les notes qu'il prend sur le terrain ${ }^{52}$ vont même dans le sens contraire: dans les bals les Noirs et les Blancs dansent ensemble (comme l'avait noté Pierson), des familles de «Blancs » incorporent en fait des Métis, ce qui dilue la «ligne de couleur», les mariages sont organisés en essayant surtout d'éviter les pauvres et des Blancs occupent aussi les positions les plus basses, comme celles de cireurs de chaussures.

Par ailleurs, dans une partie de son texte, Nogueira va clairement dans le sens de Pierson. Ainsi, il conclut qu'il n'existe pas de groupes raciaux ou de couleur séparés. Il n’y a pas à proprement parler de relations entre races mais des relations entre individus possédant différentes caractéristiques sociales dont un ton de couleur et une classe sociale. Il reconnaît aussi que la couleur n'est pas définie une fois pour toute pour chaque individu, puisque «la propre identification d'un individu en relation à la couleur dépend, jusqu'à un certain point de son association habituelle ou traditionnelle avec des groupes formés de façon prédominante par des noirs ou des blancs » (p. 202). Par ailleurs, dans les bourgades rurales, les relations se font entre gens qui se connaissent réduisant ainsi forcément sur le long terme l'effet de la couleur. Nogueira finit même par contredire son propos central: «Selon l'endroit, une personne, en raison de sa couleur noire, rencontre plus de difficultés qu'un blanc. Mais à Itapetininga 
non, parce qu'une personne qui a des relations, qui est travailleuse et honnête aura toujours de la facilité » (p. 202). L’argument principal de Nogueira est donc que les Noirs peuvent être victimes d'humiliation dans différentes situations, par exemple des disputes. Mais il ne parvient pas à montrer les conséquences sociales de ce fait. De même, il ne cherche pas plus qu'en 1942 à montrer que l'on peut séparer analytiquement classe et couleur.

Un autre aspect du sujet porte sur l'étude de la mobilité sociale (Nogueira, 1962). Notre auteur veut montrer le caractère ralenti des trajectoires des gens de couleur par rapport à celles des immigrés européens. Il constate ainsi qu’à partir des années 1930, les descendants d'Italiens commencent à accéder aux positions dominantes alors que les gens de couleur n'y parviennent pas. Le texte sous-entend, mais ne démontre pas, que la raison en est le racisme. En effet, des éléments cités par Nogueira lui-même rendent cette conclusion difficile à établir. Premièrement parce qu'un facteur évident a été la qualification professionnelle et l'esprit d'entreprise supérieurs des Italiens. La proportion des gens de couleur qui occupait une position favorable dans l'artisanat à la fin du $\mathrm{XIX}^{\mathrm{e}}$ a d’ailleurs en partie régressé. Ensuite, la compétition ethnique a semblet-il été remplacée par des alliances de classes entre Italiens et Métis. Enfin, alors que Nogueira suggère que les Européens ont été favorisés par la possibilité qu'ils ont de se marier plus facilement avec des femmes de l'élite blanche, il est incapable de dire si les mariages consacrent ou facilitent l'ascension.

Il ressort finalement de l'étude que dans ce monde rural rigide socialement, seuls parviennent à s'élever ceux qui bénéficient d'un patrimoine ou d'un réseau de relations suffisant, les autres étant toujours menacés de stagnation voire de déclin. Nogueira suggère que l'ascension est encore plus difficile pour les Noirs mais son seul argument solide tient dans la réticence du club social de l'élite à intégrer des non-Blancs. 


\section{POSTÉRITÉ DE NOGUEIRA}

L’article de Nogueira sur le préjugé de marque, écrit en accord avec Florestan Fernandes, a fait sensation à sa sortie et reste aujourd'hui un classique de la sociologie brésilienne. Pourtant cela n’a pas été suffisant pour permettre à son auteur d'obtenir une position institutionnelle lui permettant de développer ses idées. En effet, après le retour de Pierson aux États-Unis, sa propre position à l'ELSP devient moins forte (Cavalcanti, 1999), alors que celle de Fernandes, adversaire de Pierson, se consolide à l'USP après le départ de Bastide. Les années 1955-1970 seront ainsi particulièrement difficiles pour notre auteur.

Aujourd'hui, Nogueira est de nouveau apprécié, essentiellement pour avoir attirer l'attention sur le racisme dès les années 1940 et pour l'avoir étudié de manière empirique. Cette contribution a été évaluée en fonction de l'état de la sociologie brésilienne de l'époque. Ainsi certains spécialistes ont cherché à interpréter l'oeuvre de Nogueira dans le cadre de l'opposition Américains / Brésiliens. Si Degler (I97I) le voit faussement comme un disciple de Bastide, pour Guimaraes (1999) et Chor Maio (2007), Nogueira peut être rattaché à une école brésilienne qui se serait opposée au «mythe de la démocratie raciale» propagée par les Américains. Selon Chor Maio, bien que Nogueira fut en porte-à-faux vis-à-vis de certaines idées de Florestan Fernandes, il s'oppose surtout à l'idée centrale de Pierson qui voudrait que la discrimination et les préjugés contre les Noirs ne soient qu'une question de classe. Pour Guimaraes, l'apport de Nogueira consiste surtout à montrer l'effet du racisme sur la mobilité sociale et s'oppose ainsi à ses maîtres de Chicago. Seyferth (1999), de même, retient des travaux de Nogueira l'idée que la couleur et les formes physiques de la négritude sont plus que des indicateurs sociaux (idée piersonienne) mais sont le fond même du préjugé racial.

L'étude que nous avons présentée ici est plus prudente et se fonde moins sur les résultats finaux que sur la démarche. Elle part du principe que l'on ne peut comprendre Nogueira sans avoir bien lu Pierson (Cavalcanti,1999). Nous rajoutons ici l'idée, qu'il faut comprendre comment Nogueira a essayé de d'interpréter les relations raciales en vivant et étudiant aux États-Unis. Cette 
expérience a certainement permis à cet auteur de saisir mieux que d'autres la sociologie de Chicago, tout en percevant différentes facettes du «racisme» et en prenant en compte une plus large gamme de faits.

Il nous semble donc impossible de rattacher complètement Nogueira à une sociologie brésilienne anti-raciste, telle que celle initiée par Bastide. La sociologie de Nogueira reste en effet, à sa manière, assez américaine. Nogueira s'éloigne de Pierson sur certains points mais il en est proche sur d'autres. En fait, il hésite et balance. Il voit bien à l'instar de Pierson que l'on n'a pas affaire à des groupes raciaux en conflit. En même temps, il ne croit pas judicieux de limiter l'étude de la «situation raciale» à l'historique de la rencontre entre les peuples. Le sociologue, selon lui, doit dénoncer le statu quo racial. En cela, il est très fidèle aux positions des jeunes praticiens des sciences sociales au Brésil dans les années 1940-1950. Par ailleurs, il ne peut accepter de résoudre idéologiquement les problèmes scientifiques. Il veut montrer les choses par un lent travail de terrain, comme ses maîtres américains. C'est là que l'on perçoit chez lui un conflit latent entre l'usage de ses données et l'intention militante. Ses différents textes apportent des éléments montrant indubitablement que le statut social de la négritude est bas et que cela peut placer les personnes de couleur dans des situations délicates. Il montre aussi que la vie courante dans l'état de São Paulo peut conduire les Noirs à sentir ou vivre des opinions racistes. Comme le note justement Cavalcanti (1996), il montre la plasticité des préjugés et leurs liens avec le milieu qui n’est pas celui d’une conséquence automatique d'une certaine structure sociale. Mais il ne réussit pas pourtant à aller plus loin dans la dénonciation des effets de ce racisme. Il ne parvient pas non plus à séparer mépris de classe et mépris racial. Si l’on retient le fait que l'essentiel de ce qui vient d'être évoqué se trouve déjà en grande partie dans les textes de Pierson, la singularité de notre auteur tient plutôt à ses intentions et au conflit, chez lui non résolu, entre militantisme et scientisme strict.

Le cas Nogueira représente en outre, pour l'histoire de la sociologie, un exemple intéressant de défi ambivalent par rapport au maître. Le Brésilien garde la méthode et certains concepts de son «mentor». Mais il modifie l'objectif et certaines définitions (la «situation raciale» par exemple ou la notion de 
«problème racial»). Il insiste sur un point ignoré de Pierson pour des raisons qui vont contre l'idéologie scientifique qu’on lui a enseignée; mais en même temps il le confirme plus ou moins volontairement sur d'autres points. Il ne parvient pas à s'éloigner de Pierson autant qu'il le voudrait ne serait-ce qu'en raison de l'importance accordée à la pratique de l'induction après fieldwork prolongé. Mais un élément d'explication terre à terre doit aussi être évoqué : la position institutionnelle. Tant qu'il travaille avec Pierson, Nogueira reste prudent dans la dénonciation du racisme. Au début des années 1950, alors qu’il se rapproche de Bastide, il est plus virulent. Puis, allant de poste en poste, il cherche une formule originale qui lui permette de concilier ses buts contradictoires. Cependant, en dépit des aléas de sa vie professionnelle, Nogueira n’a pas dévié de son objectif: faire un compte rendu fondé et prudent sur la situation des Noirs dans les environs de São Paulo. Sa dernière publication d'envergure (Nogueira, 1992) consiste d’ailleurs en la biographie d’un politicien local à la peau noire.

Pour conclure, si l'on passe en revue les critères d'analyse utilisés dans ce texte, la biographie des auteurs en matière d'études des relations raciales joue donc sans aucun doute un rôle non négligeable, de même que la formation universitaire et les idées politiques. Mais on peut également souligner le rôle des relations de disciple à maître, les méthodes d’enquête et la position institutionnelle. Dans un sujet aussi miné politiquement, les conclusions des sociologues sont rarement le résultat évident d'un recueil impartial des faits mais plutôt une combinaison subtile d'intentions idéologiques, d'impératifs de carrière et de dispositifs méthodologiques.

\section{BIBLIOGRAPHIE}

BACELAR J., 200I, Negros e brancos em Salvador, Rio de Janeiro, Pallas.

BASTIDE R., 1939, «État actuel des études afro-brésiliennes», Revue Internationale de Sociologie, 47, (I-2), p. 77-89. 
BASTIDE R., 1943, «Introdução ao estudo de alguns complexos afro-brasileiros », Revista do Arquivo Municipal, 90, p.7-54.

BASTIDE R., 1950, «Rêves de Noirs», Psyché, (49), p. 802-8II.

-, et FERNANDES, F., 1955), Brancos e negros em São Paulo, São Paulo, Unesco / Anhembi.

CANDIDO A., 1959, «A sociologia no Brasil » in Enciclopedia Delta-Larousse, Rio de Janeiro, Delta.

-, 1987, A educação pela noite e outros ensaios, São Paulo, Ática.

CARDOSO I., 1987, «Entrevista com Roger Bastide », Discurso, (16), p . |81-197.

CAYTON H. et DRAKE, St. C., 1945, Black Metropolis: A Study of Negro Life in a Northern City, New York, Harcourt Brace.

CAVALCANTI M. L., 1995, «Oracy Nogueira: esboço de uma trajetória intelectual», História, Ciências, Saúde - Manguinhos 2, (2), p. I19-134.

-, 1996, «Oracy Nogueira e a Antropologia no Brasil: O estudo do estigma e do preconceito racial », Revista Brasileira de Ciências Sociais, II, (31), p. 5-28.

-, 1998, «Apresentação » in Preconceito de Marca: as relações raciais em Itapetininga, São Paulo: EDUSP.

-, 1999, «Preconceito de marca, etnografia e relações raciais », Tempo Social, II, (I), p. 97-110.

CHACON V., 1993, Gilberto Freyre: uma biografia intellectual, Recife, Editora Massangana. CHAPOULIE J.-M., 200I, La tradition sociologique de Chicago, 1892-1961, Paris, Le Seuil. CHAPOULIE J.-M., 2002, «La tradition de Chicago et l'étude des relations entre les races», Revue européenne des migrations internationales, 18, (3), p. 9-24.

CHOR MAIO M., 1997, A história do Projeto UNESCO Estudos raciais e ciências sociais no Brasil, Thèse de doctorat, Universidade federal do Rio de Janeiro. -, 2005, «From Bahia to Brazil. The UNESCO race relations projects », in J. Souza and V. Sinder, (eds.), Imagining Brazil, Lanham, MD, Lexington Books. -, 2007, «O Racismo no microscópio: Oracy Nogueira e o projeto UNESCO », Estudios Interdisciplinarios de América Latina y el Caribe, 19, (I), p. 35-52. 
COELHO R., 1981, «Depoimento », Lingua e Literatura, (10-13), p. I21-133.

CORRÊA M., 1998, As ilusões da liberdade: a Escola Nina Rodrigues e a antropologia no Brasil, Bragança Paulista, Edusf.

CRUZ L., 2003, «Democracia racial: uma hipótese », in F. Quintas (org.) Evocações e interprétações de Gilberto Freyre, Recife, Massangana.

DEGLER C., 197I, Neither Black nor White, New York, MacMillan.

DENIS P., 1909, Le Brésil, Paris, Armand Colin.

FRAZIER F., 1936, «Review of Formação do povo brasileiro by Oliveira Viana », American Journal of Sociology, 4I, (5), p.674-675.

FREYRE G., 1933, Casa grande e senzala, São Paulo, Companhia editora nacional.

GUIMARAES A. S., 1996, «Cor, Classes e Status nos Estudos de Pierson, Azevedo e Harris na Bahia: 1940-1960 », in M. Chor Maio, R. V. Santos, (eds) Raça, ciência e sociedade, Rio de Janeiro, Fiocruz.

-, 1999, «Resenha de preconceito de marca 》, Revista Brasileira de Ciências Sociais 14, (4I), p. 169-17|.

HERSKOVITS M., 1925, «The color line », American mercury 6, (22), p. 204-208.

JACKSON L. C., 2007, «Gerações pioneiras na sociologia paulista (1934-1969) », Tempo social, 19, (I), p. II5-130.

KANTOR I. MACIEL D., SIMOES J., 200I, A escola livre de sociologia e política. Anos de formação 1933-1953, São Paulo, Escuta.

LIMONGI F. P., 1989, A Escola Livre de Sociologia e Política em São Paulo, in S. Miceli (ed.), História das Ciências Sociais no Brasil (vol. I). São Paulo, Vértice/ldesp/ Finep.

MICELI S., 1987, «Condicionantes do desenvolvimento das ciências sociais no Brasil 1930-1964 », Revista brasileira de ciências sociais, 2, (5), p. 5-26.

MENDOZA E., 2005, «Donald Pierson e a escola sociologica de Chicago no Brasil: Os estudos urbanos na cidade de São Paulo (1935-1950) ». Sociologias, (14), p. 440-470. 
NASCIMENTO E. A. D., 200I, Dance of deception: a reading of race relations in Brazil, in C. Hamilton (ed.), Beyond racism. Boulder (Col.), Rienner.

NOGUEIRA O., 1942, «Atitude desfavorável de alguns anuciantes de São Paulo em relação aos empregados de cor », Sociologia 4, (4), p. 328-358.

-, 1947, «Review of Slave and Citizens: The Negro in the Americas by Frank Tannenbaum », American Journal of Sociology, 53, (1), p. 70-72.

-, 1950, «Vozes de Campos do Jordão: experiências sociais e psíquicas de tuberculoso pulmonar no estado de São Paulo », Sociologia, São Paulo.

-, 1955a, «Preconceito de marca e preconceito racial de origem », Anhembi, 18, (53), p. 279-299.

-, 1955b, Relações raciais no municipio de Itapetininga, in R. Bastide, F. Fernandes, Brancos e negros em São Paulo, São Paulo, Unesco/Anhembi.

-, 1962, Família e comunidade: um estudo sociológico em Itapetininga, Rio de Janeiro, Centro Brasileiro de Pesquisas Educacionais.

- ,1964, Pesquisa social: introdução ás suas técnicas, São Paulo, Ed. Nacional.

-, 1992, Negro político, político negro: A vida do Dr. Alfredo Casemiro da Rocha parlamentar da república velha, São Paulo, Edusp.

-, 1995, «Depoimento », História, Ciências, Saúde - Manguinhos, 2, (2), p. I22-I34.

- 1998, Preconceito de Marca: as relações raciais em Itapetininga, São Paulo, EDUSP.

OLIVEIRA VIANNA F. J., 1932, Raça e assimilação, São Paulo,

Companhia Editora Nacional.

PIERSON D., 1942, Negroes In Brazil: A study of race contact at Bahia, Chicago, University of Chicago Press.

-, 1945, Brancos e Pretos na Bahia, São Paulo, Companhia Editora Nacional.

-, 1951, «O preconceto racial segundo o estudo de "situação racial" »», Sociologia, 13 (4), p. 305-324.

RAMOS A., 1934, O negro brasileiro, São Paulo, Companhia Editora Nacional.

-, 1943, Guerra e Relações de Raça, Rio de Janeiro, Departamento Editorial da União Nacional dos Estudantes. 
RAMOS, J de S., 2003, «Ciência e racismo: uma leitura crítica de Raça e assimilação em Oliveira Vianna », História, Ciências, Saúde-Manguinhos, 10 (2), p. 573-60I.

SCHWARCZ L. M., 2003, «Not black, not white: just the opposite. Culture, race and national identity in Brazil », Working paper $n^{\circ}$ CBS-47-03, Centre for Brazilian Studies, University of Oxford.

SEYFERTH G., 1999, «Resenha de preconceito de marca », Mana 5, (I), p. 157-I80.

SCHWARTZMAN S., 1979, Formação da comunidade cientifıca no Brasil, Rio de Janeiro, Ed. Nacional.

VALLADARES L., 2006, A Escola de Chicago e o Brasil, Belo Horizonte, Editora UFMG.

VELHO G., 2008, «Gilbero Freyre: trajtoria e singularidade », Sociologia, problemas e praticas, (58), p. II-2I.

VIANNA F. J., 1932, Raça e assimilação, Rio de Janeiro, Companhia Editora Nacional.

VILA-NOVA S., 1998, Donald Pierson e a Escola de Chicago na Sociologia brasileira: entre humanistas e messiânicos, Lisboa, Vega Universidade.

WILLEMS E., 1940, Assimilação e populações marginais no Brasil, São Paulo, Companhia Editora Nacional.

-, 1947, Cunha, tradição e transição em uma cultura rural do Brasil, São Paulo, Secretaria da Agricultura.

- ,1949, «Racial attitudes in Brazil », American Journal of Sociology, 54, (5), p. 403-409.

-, 1955, «Resenha de Populações meridionais do Brasil por F. J. Oliveira Viana », The Hispanic American Historical Review, 35, (2), p. 288-290.

-, 1957, «Resenha de Evolução do povo brasileiro por F. J. Oliveira Vianna », The Hispanic American Historical Review, 37 (4), p. 499-500. 